\title{
PATRIMONIALIZACIÓN, DESARROLlO TERRITORIAL Y NUEVOS MODELOS DE GOBERNANZA. EL CASO DEL RAMAL FERROVIARIO TALCA-CONSTITUCIÓN EN la Región del Maule, Chile
}

\author{
Fabiola Leiva Cañete* \\ Pablo Díaz Meeks**
}

\section{Resumen}

A partir de la caracterización del caso del ramal Talca-Constitución, monumento histórico propiedad de una empresa pública de gestión autónoma y último ramal ferroviario en funcionamiento en Chile, el artículo expondrá algunas reflexiones en torno a la multidimensionalidad que deben asumir los procesos de patrimonialización en contextos donde conviven expresiones culturales y naturales únicas con dinámicas de pobreza, exclusión, desigualdad y riesgo ambiental. También se discutirán los aportes que en este escenario tienen las miradas que buscan armonizar los procesos socioculturales de reivindicación y apropiación del territorio y su dotación de recursos bioculturales, con el diseño de estrategias de desarrollo territorial que colaboren con el bienestar y la calidad de vida de las comunidades. Finalmente, se abordará la necesidad de imaginar y testear nuevos acuerdos de gobernanza y sistemas de gestión que se hagan cargo de la complejidad territorial, por un lado,

* Máster en Gestión de Políticas Culturales y Desarrollo, Cátedra Unesco-Universidad de Girona. Investigadora principal de Rimisp-Centro Latinoamericano para el Desarrollo Rural, Santiago (Chile). [fleiva@rimisp.org; fleivacanete@gmail.com]; [https://orcid.org/0000-0003-3425-6832].

** Psicólogo, Universidad de Valparaíso; cursando maestría en Desarrollo Humano en Flacso, Argentina. Habitaciones Comunes, Valparaíso (Chile). [pdiazmeeks@gmail.com]; [https://orcid.org/0000-0002-4534-8671].

Recibido: 24 de junio de 2019 / Modificado: 12 de agosto de 2019 / Aceptado: 20 de agosto de 2019

Para citar este artículo

Leiva Cañete, F. y Díaz Meeks, P. (2020). Patrimonialización, desarrollo territorial y nuevos modelos de gobernanza. El caso del ramal ferroviario Talca-Constitución en la región del Maule, Chile. OPERA, 26, 37-53.

Dor: https://doi.org/10.18601/16578651.n26.04 
$y$, por otro, puedan responder a los nuevos estándares de participación que diversos grupos sociales exigen en la actualidad.

Palabras clave: patrimonio; gobernanza; desarrollo territorial; comunidades.

\section{PATRIMONIALIZATION, TERRITORIAL DEVELOPMENT AND NEW GOVERNANCE MODELS. THE CASE OF THE TALCA- CONSTITUCIÓN RAILWAY BRANCH IN THE MAULE REGION, CHILE}

\section{Abstract}

Starting from the characterization of the case of the railway branch Talca-Constitución - a historical monument owned by a public company of autonomous management and the last railway branch still operating in Chile - this article will present some reflections on the multidimensionality that should be assumed by the patrimonialization processes in contexts of conflict, where unique cultural and natural expressions coexist with indicators of poverty, exclusion, inequality and environmental risk. Secondly, we will focus on the contributions of adopting an approach that, in such a scenario, seeks to harmonize the sociocultural processes of claiming and appropriation of the territory and its endowment of biocultural resources, with the design of territorial development strategies that help improve welfare and quality of life of the communities.
And finally, we emphasise the need to imagine and test new governance agreements and management systems that, on the one hand, take into account the territorial complexity, and on the other, can respond to the new standards of participation currently demanded by the diverse social groups involved.

Key words: Heritage; governance; territorial development; communities.

\section{INTRODUCCIÓN}

Las reflexiones que se presentan a continuación son el resultado de diversas iniciativas llevadas a cabo en la región del Maule, en Chile, que de manera directa, y en otros casos de forma más periférica, nos permitieron construir una visión diagnóstica con enfoque territorial del ramal Talca-Constitución ${ }^{1}$, el último ramal ferroviario vigente y operativo en Chile. La mayoría de estas acciones, algunas de investigación y otras de asesoría, realizadas en el marco del programa Desarrollo Territorial con Identidad Cultural (DT-IC) (RIMISP, s. f.) de Rimisp-Centro Latinoamericano para el Desarrollo Rural, y otras más recientes, se desarrollaron a petición de entidades públicas de nivel central, fundamentalmente asociadas a la institucionalidad relativa a los temas de patrimonio cultural y agricultura familiar campesina en Chile.

A partir de este diagnóstico territorial, esto es, de una caracterización multidimen-

\footnotetext{
1 Han contribuido en estos procesos de investigación Carlos Pallacán, geógrafo, magíster en Desarrollo Rural, Universidad Austral, y Francesca Compagnone, máster en Economía del Desarrollo de la Universidad de Florencia.
} 
sional y multiactoral del espacio socialmente construido en torno al tren y sus dinámicas, y teniendo a la vista algunos elementos de contexto que describen la condición y el estado del ramal en la actualidad, a saber: 1) que el ramal ferroviario Talca-Constitución es un bien de propiedad de la Empresa de Ferrocarriles del Estado (EFE), empresa pública de gestión autónoma ${ }^{2}$; 2) que el estado de conservación de las estaciones y el tren, componentes del sistema Ramal Talca-Constitución, declarado Monumento Histórico, presenta un deterioro extremo y acumulativo que amenaza, no solo la integridad y los valores patrimoniales, sino también la calidad del único servicio de conectividad con que cuentan algunas comunidades del territorio; 3) que el tren continúa en funcionamiento, fundamentalmente gracias a un subsidio entregado por el Estado, a través del Ministerio de Transportes, precisamente por la condición de aislamiento en que viven algunas localidades; 4) que, además, en el territorio se constata la presencia de amenazas naturales y antrópicas significativas, algunas de ellas asociadas a la acción de grandes empresas, propondremos algunas reflexiones en torno a los desafíos que enfrentan las comunidades, el Estado y algunos actores extraterritoriales involucrados con el ramal respecto de la conceptualización, gobernanza y gestión del territorio que este eje ferroviario articula sistémicamente.

La primera reflexión que se planteará tiene relación con el enfoque de patrimonialización que amerita la situación del ramal, desde una lógica territorial, biocultural, de desarrollo y de ejercicio de derechos. La segunda se refiere a los procesos de reconocimiento y apropiación de las comunidades en torno a su patrimonio cultural y natural, y los aportes que este proceso de puesta en valor puede tener en términos de activación de economías locales, cohesión social y resiliencia socioambiental. Finalmente, la tercera reflexión apuntará a los desafíos del diálogo territorial entre actores diversos, internos y externos, públicos y privados, y la necesidad de innovaciones en torno a procesos de gobernanza y gestión de espacios o recursos fuertemente reivindicados por comunidades locales.

\section{EL RAMAL FERROVIARIO TALCA-CONSTITUCIÓN}

Las ferrovías y sus complejos, denominadas ramales, son parte de la historia ferroviaria de Chile, país en extremo longitudinal, con 8.500 kilómetros de vías. Estas dotaron de accesibilidad al país y generaron una efectiva integración territorial que impactó el desarrollo económico y social fortaleciendo la conectividad geográfico-económica, mejorando la seguridad y el control de los espacios, e incidiendo en el surgimiento de localidades y ciudades (Morales, 2013).

Los ramales tuvieron su apogeo en Chile a inicios del siglo xx, y declinaron hasta prácticamente desaparecer en las dos últimas décadas

2 Empresas creadas por ley, del Estado o sociedades, en que este tiene una participación accionaria superior al $50 \%$ o designa a la mayoría de los miembros de su directorio. 
del mismo siglo, fundamentalmente ante el desarrollo y la competencia de otros medios de transporte. El ramal entre la ciudad de Talca y el puerto de Constitución, en la región del Maule, fue uno de cinco construidos y operados, su instalación se llevó a cabo entre los años 1888 hasta 1915 , conectando estaciones y parajes a lo largo de 90 kilómetros de vías.

El ramal Talca-Constitución incidió significativamente en el tráfico de mercancías agroganaderas con mercados mayores, nacionales e internacionales, y la sustitución del flujo fluvial en el río Maule (Morales, 2013). La agricultura y el comercio incipiente ocasionaron el surgimiento de pueblos y ciudades a orillas de caminos, ríos y esteros que, junto a la concurrencia de los campesinos, facilitaron el poblamiento de la zona.

Actualmente, el ramal Talca-Constitución es el último representante en funcionamiento de los ramales para pasajeros del contexto rural del país, el cual en 2015 cumple 100 años como servicio de transporte operativo e hito del patrimonio ferroviario e industrial de Chile. Fue declarado monumento histórico el 25 de mayo de $2007^{3}$.

\section{Actores clave}

Como se señaló, el ramal es un bien de propiedad de la Empresa de Ferrocarriles del
Estado (EFE), y es operado por unas de sus filiales ${ }^{4}$. La privatización de las empresas estatales tuvo su origen en la política de privatización y desregulación iniciada por la dictadura militar entre los ańos 1973 y 1989, aunque el proceso tuvo continuidad con los primeros gobiernos democráticos de transición. Es el caso de la Empresa de Transporte Ferroviario (Ferronor), privatizada durante el gobierno de Frei Ruiz-Tagle a través de la Ley 19.170 de 1992, creando con ello la Empresa de Ferrocarriles del Estado (EFE). La definición de empresa pública es particularmente difícil en el derecho chileno porque las distintas empresas que existen obedecen a regímenes jurídicos, reglas y funciones diferentes, pero en general cumplen las siguientes características: 1) son entidades creadas por ley; 2) tienen personalidad jurídica de derecho público propia y patrimonio propio; 3) las empresas públicas forman parte de la Administración del Estado; 4) se trata de entidades autónomas, pero que se encuentran sujetas a la supervigilancia de la Administración; 5) las empresas del Estado, al formar parte de la Administración, se encuentran sometidas al principio de legalidad y de probidad administrativa, así como a los controles de la Cámara de Diputados, la Contraloría General de la República y el Consejo para la Transparencia (von Lobenstein, 2016). En lo que se refiere a la política de administra-

3 La declaratoria, que atiende la ley de Monumentos en Chile, incluye la vía férrea estructurante de $88 \mathrm{~km}$, faja vía, diversas estaciones, puentes, tornamesas, un túnel y obras de arte. Se suman 4 unidades rodantes de Buscarril para pasajeros y carga liviana. El total de superficie protegida alcanza las 75,22 ha. Se destacan el modernismo de las estaciones de Talca y Constitución, ambas en terminales extremos y con obras de reconstrucción durante el siglo xx. Las estaciones intermedias destacan por su arquitectura chilena tradicional. El puente ferroviario Banco Arena posee estructura metálica y diseńo de Gustav Eifel.

4 https://www.trencentral.cl/buscarril 
ción y gestión del ramal, EFE se ha conducido precisamente en coherencia con esta condición de autonomía y subsidiariedad, respondiendo con los estándares mínimos exigidos para un servicio de transporte público, y teniendo una participación no obstructiva, aunque en extremo pasiva en lo que se refiere a las diversas iniciativas, tanto públicas como privadas, que han intentado posicionar y visibilizar su valor histórico y patrimonial.

Por su parte, las comunidades locales, especialmente las que utilizan el tren, han sido fundamentales en su incansable lucha por conservar el patrimonio cultural asociado al eje ferroviario, y por demandar mejoras en las condiciones de calidad del servicio. Se trata de comunidades campesinas, algunas en condición de aislamiento y, en general, afectadas por una alta dispersión territorial, muy pequeñas en población y atravesadas por las dinámicas de migración campo-ciudad, fundamentalmente en lo que se refiere a la composición etaria, con predominio de adultos mayores. Por otro lado, la adhesión que despierta el ramal incluye también a diversas organizaciones regionales y extraterritoriales de "amigos de los trenes", que constantemente difunden información y noticias relativas al último ramal en vigencia, al mismo tiempo que operan en algunos casos como asesoría profesional al servicio de los actores locales.

El Estado, por su parte, a través de su institucionalidad central vinculada a la salvaguardia y protección del patrimonio cultural, así como a través del gobierno regional del Maule, ha apoyado diversas iniciativas de puesta en valor del monumento histórico, algunas con un enfoque más conservacionista, otras con énfasis productivos asociados a las oportunidades turísticas que ofrece el bien y el paisaje que lo acoge (Casals y Errázuriz, 2013). Sin embargo, hasta la fecha, no ha abordado concretamente su doble condición de patrimonio histórico y medio de transporte, ni la gobernanza de dicho bien público y su gestión, actualmente limitada a criterios de maximización de rentabilidad económica.

Finalmente, entre los actores clave del territorio se debe mencionar a un conjunto de empresas, grandes y medianas, vinculadas a la industria forestal, por un lado, y a la agroindustria por otro, incluido el cultivo de vides en gran escala, cuyo efecto territorial, ambiental y social es de gran impacto.

\section{DESIGUALDADES Y PATRIMONIO EN EL TERRITORIO DEL RAMAL}

En la trayectoria de trabajo sobre desigualdades y desarrollo inclusivo con cohesión territorial que tiene Rimisp, y en particular sus experiencias en la implementación del programa Desarrollo Territorial con Identidad Cultural (DT-IC) durante una década, se ha venido constatando la presencia de territorios, particularmente rurales, con una alta dotación de recursos naturales, biodiversidad y patrimonio cultural que, al mismo tiempo, enfrentan múltiples niveles de conflictos, desigualdad y exclusión. Los territorios que recorre y organiza el ramal Talca-Constitución no son ajenos a esta paradoja.

Para efectos de realizar una caracterización multidimensional de los territorios, y especialmente de las comunidades que habitan el eje que recorre el ramal Talca-Constitución, se definió un "territorio" que incluye 55 locali- 
dades adyacentes al eje ferroviario, que mantienen una relación directa e indirecta con el bien en términos de sistema de conectividad y elemento de articulación sociocultural. Esta suerte de área de influencia involucra cinco municipios (Talca, Maule, Pencahue, San Javier y Constitución) y una población cercana a las 12 mil personas.

En términos demográficos, las localidades presentan baja densidad poblacional y la distribución por rangos etarios arroja porcentajes altos de población de adulto mayor, especialmente en las comunas de Pencahue y San Javier.

Las actividades agrícolas, ganaderas, de caza y silvicultura concentran el mayor porcentaje de personas en condición de ocupación en esta área, destacándose el caso de Pencahue, donde cerca del $80 \%$ de la población rural se desempeña en esta categoría de actividades. A medida que nos acercamos a los polos urbanos de Talca y Constitución se evidencia una mayor heterogeneidad en los sectores de ocupación.

Respecto a la situación de pobreza, los resultados de la encuesta Casen (2017) evidencian una situación muy preocupante, especialmente en las áreas rurales donde más de un cuarto de la población se encuentra en condición de pobreza por ingresos y más de la mitad en pobreza multidimensional. Asimismo, el $44 \%$ de la población rural de las comunas de Pencahue y el 39,4\% de Maule se encuentran en condiciones de pobreza multidimensional, porcentajes que representan el doble de la media del país, que se acerca al $21 \%$.

En términos globales, se puede observar que la tasa de pobreza multidimensional en el área de influencia del ramal se acerca al $40 \%$, por arriba del promedio nacional $(37,4 \%)$ y regional $(32,4 \%)$. Es más, el $11,8 \%$ de la población rural de las comunas que recorre el ramal vive en condición de pobreza por ingresos, frente al 8,6\% de la población nacional que se encuentra en la misma situación. Finalmente, es relevante destacar que las desigualdades de género parecen sumarse a las brechas territoriales, tal como evidencian las cifras relativas a los indicadores de pobreza multidimensional en las áreas rurales vis a vis las urbanas, en el caso de hombres y mujeres.

En lo que se refiere a indicadores para educación, la mayoría de la población que habita las localidades ligadas al ramal cuenta con un nivel de educación básica o media, representando un 82,6\% del total de la población.

Por su parte, es posible identificar que la mayoría de la red vial que se distribuye y conecta a las localidades del sector circundante al ramal posee suelo natural y ripio en algunos sectores. Los tramos que cuentan con pavimento o pavimento básico por lo general son aquellos cercanos a las áreas urbanas de Talca y Constitución. Por su parte, la Subsecretaría de Desarrollo Regional y Administrativo (subDE$\mathrm{RE}$ ) identifica a las localidades de Maquehua, Huinganes, Forel, Los Boquis, Los Romeros, Las Lisas, Quebrada Pichamán y Pichamán en Constitución y El Alto en San Javier, bajo la condición de localidades aisladas 5 .

5 Según la definición del Ministerio de Obras Públicas y Vivienda: "Las zonas aisladas son zonas de habitabilidad condicionada por factores geográficos, por una parte, y de escasa infraestructura de conexión por el otro lado. 
Además de levantar indicadores económicos y sociodemográficos, ha sido relevante, en una mirada multidimensional, levantar variables naturales del territorio. Esta imagen expone una situación preocupante en términos de las amenazas naturales y antrópicas a las que se exponen los territorios y las comunidades del ramal. Amenazas naturales como la disminución de las precipitaciones, la erosión y los eventos sísmicos; y otras de carácter antrópico como los incendios forestales, la extracción de áridos, la agricultura de gran escala y, particularmente, los impactos de la explotación que realiza la industria forestal, evidencian una situación de alto riesgo para el tren, sus instalaciones y, por supuesto, para las comunidades que habitan los territorios que une, así como una fuerte presión ambiental a la que estás expuestas la vegetación endémica y los cursos fluviales, con todo el impacto ecosistémico que dicha alteración puede desencadenar.

El conjunto de estos datos construidos a partir de fuentes secundarias y la percepción de las comunidades, revela una condición de desigualdad territorial significativa que afecta a los habitantes del ramal. La desigualdad territorial significa que el lugar donde nace y crece una persona tiene una influencia significativa en determinar quién es y qué llegará a ser (Rimisp, 2018). Tal condición no solo es moralmente inaceptable, sino que también es un obstáculo para el desarrollo general de un país. Por su parte, la cohesión territorial es una condición por la cual todos los territorios de un país pueden expresar su potencial de desarrollo y ninguno de ellos es persistentemente marginado. La desigualdad territorial es la ausencia de cohesión territorial (Rimisp, 2018).

Finalmente, cabe destacar que estas desigualdades territoriales se acentúan al intersectar con otros sistemas de exclusión y subordinación, en el caso del ramal, particularmente las desigualdades de género. Esta mirada, conocida como enfoque interseccional, es clave para ampliar el análisis de las desigualdades al poner de manifiesto los efectos complejos, irreductibles y variados que adquieren según la intersección de diferentes sistemas de exclusión (Brah y Phoenix, 2004).

Respecto a la presencia de diversos elementos de valor patrimonial, el proceso de identificación, sistematización y registro de distintas fuentes da cuenta de una vasta producción cultural alrededor del ramal TalcaConstitución, que revela la importancia que tiene este bien patrimonial y el sistema cultural que articula, en la memoria colectiva y la identidad, no solo de las comunidades del territorio, sino también de actores extraterritoriales.

En cuanto al patrimonio cultural material, sin duda la presencia del servicio ferroviario del ramal Talca-Constitución, declarado monumento histórico, constituye el hito principal en torno a bienes patrimoniales monumentales de relevancia. De todas maneras, y sin perjuicio de lo anterior, existe al menos una treintena de bienes patrimoniales monumentales en el territorio, entre los que se destaca el Santuario Inmaculada Concepción de Corinto, que cuenta con la denominación

Es decir, no se sabe qué es primero, si la poca habitabilidad es por la falta de infraestructura, o que por la baja habitabilidad la infraestructura no se justifica” (Ministerio de Obras Públicas y Vivienda, 2011). 
de Monumento Nacional en la categoría de Monumento Histórico, otorgada el año 2007 (CMN, s. f.).

Desde sus inicios, el ramal Talca-Constitución se constituye en un eje articulador, no solo desde el punto de vista económico, como sistema de transporte y distribución de diversas mercancías, sino especialmente como elemento constructor de un paisaje cultural que se conforma a partir de asentamientos en torno a estaciones y paraderos, cuyas prácticas sociales se estructuran alrededor del funcionamiento del tren. Tal es su impacto como nuevo elemento constructor de la vida social de los territorios que recorre, que con él desaparece el modo de vida asociado al transporte fluvial que caracterizaba la zona hasta principios del siglo xx (Morales, 2013).

El ramal, a pesar de las evidentes transformaciones a las que se ha visto expuesto, en tanto tren con atributos patrimoniales que todavía se halla en funcionamiento, conserva en torno a sí una dinámica cultural específica que incluye formas de sociabilidad, tradiciones campesinas, oficios, conocimientos tecnológicos ferroviarios, relatos e incluso registros lingüísticos y comunicacionales específicos, que conforman un modo de vida singular y de valor excepcional, organizado y dependiente de la dinámica ferroviaria del ramal.

En esta línea, entre los acervos culturales producidos en torno al ramal se destaca un rico patrimonio alimentario, que incluye prácticas productivas, productos, artesanía utilitaria y preparaciones, expresiones materiales e inmateriales de un modo de vida rural campesino, que en el caso de las comunidades que ha unido el ramal en su centenaria historia, exhibe un conjunto de dinámicas con el sello del tren como factor organizador.

También encontramos, a lo largo de prácticamente todo el trayecto del tren, incluso en los territorios más afectados por el cultivo forestal, la plantación de viñedos y la producción de vino artesanal, especialmente de la cepa conocida como "cepa país", llegada a Chile con las misiones españolas en el siglo Xvi. Muchas de estas vides, según los propios ramalinos que las cultivan, son plantas centenarias que han heredado de generación en generación. Se trata de un fenómeno cultural, no solo muy arraigado en la identidad territorial de las comunidades del ramal, sino también muy extendido. Dicho en otras palabras, "todo el mundo hace vino", lo que convierte a esta práctica de alto valor cultural en un elemento axial del patrimonio del territorio.

Finalmente, pero no menos importante, especialmente si tomamos en cuenta las amenazas naturales y antrópicas que afectan el territorio del ramal reseñadas, encontramos dos zonas que presentan remanentes de un patrimonio natural endémico que se hace indispensable conservar y proteger. La zona de Pichamán fue reconocida por la Corporación Nacional de Medio Ambiente (Conama) mediante su Estrategia y Plan de Acción para la Biodiversidad en la región del Maule el año 2002, como Sitio Prioritario, debido a la presencia de bosques de hualo, integridad de ecosistemas y diversidad de comunidades biológicas. Por su parte, se constata la presencia de un área de alto valor para la conservación, denominada Bosque Maulino con ruiles y pitaos de Quivolgo, iniciativa de conservación privada administrada por la empresa forestal 
Arauco, que se ubica al interior de los predios en los cuales realiza la actividad silvícola.

\section{PATRIMONIALIZACIÓN, TERRITORIO Y DESARROLLO}

Las tendencias actuales de la praxis patrimonial pasan por la inevitable reconsideración de los territorios en los que se ubican los bienes culturales. Sin esta premisa, ni su preservación, gestión o aprovechamiento como recurso social están asegurados. Esta evolución en la discusión sobre los procesos de patrimonialización ha transitado desde una intención preferentemente estética y conservacionista, al protagonismo del desarrollo territorial. Ello supone un reforzamiento de las facetas económicas y sociales de los paisajes, entendidos estos como marcos vitales, espacios para el disfrute ciudadano y activos para el desarrollo.

Las características “territoriales” del ramal Talca-Constitución, que lo sitúan en el eje conceptual que describen nociones como las de paisaje cultural o itinerario cultural ${ }^{6}$, así como el estado que presentan el monumento histórico, el territorio, y especialmente las comunidades a las que sirve como sistema de conectividad, plantean la necesidad de una mirada integral del patrimonio que, por una parte, se haga cargo de la dimensión "biocultural" de la actividad humana, esto es, la forma en que las comunidades "interactúan con su propio ecosistema local, y con la combinación de paisajes y sus respectivas biodiversidades contenidos en ellos, de tal suerte que el resultado es una compleja y amplia gama de interacciones finas y específicas" (Toledo y Barrera-Bassols, 2008); $y$, por otro, ponga atención en la manera en que las políticas de patrimonialización pueden facilitar y potenciar procesos de ordenación del espacio y desarrollo territorial, buscando el mejoramiento de las condiciones de bienestar y calidad de vida de las comunidades y los territorios que han producido y sostenido el valor y las condiciones por las que se valora determinada expresión humana.

Desde esta perspectiva, parece relevante asumir un enfoque que ponga como eje de su análisis a la cultura y el patrimonio en su relación con la dimensión territorial, óptica que permitiría un abordaje capaz de incorporar de manera compleja las diversas dimensiones involucradas. Un enfoque territorial de esta índole puede ser definido como una vocación por interpretar la realidad a partir del análisis de la diversidad de formas que ella adquiere a escala de los territorios, y diseñar iniciativas de acción pública, no únicamente estatales, que promuevan que los territorios expresen su

6 Un paisaje cultural es una obra de arte artificial, construida por el hombre, a partir de un espacio natural. La Unesco lo define como "la representación combinada de la labor de la Naturaleza y el Hombre". Mientras que, en el Convenio Europeo del Paisaje del Consejo de Europa del año 2000, se define "como el resultado de la acción y la interacción de factores naturales y/o humanos". Por su parte, según el Comité Científico Internacional de Itinerarios Culturales del Consejo Internacional de Monumentos y Sitios (cIIC-Icomos), un itinerario cultural es una vía de comunicación terrestre, acuática, mixta o de otra naturaleza, físicamente determinada y caracterizada por tener su propia y específica dinámica y funcionalidad histórica, manifiesta en intercambios continuos multidimensionales y recíprocos de personas, bienes, ideas, conocimientos y valores en el interior de un país o entre varios países y regiones durante un periodo significativo de tiempo. 
potencial y que se reduzcan las desigualdades territoriales.

Otra óptica clave a la hora de buscar que los procesos de puesta en valor del patrimonio se alineen con una perspectiva de desarrollo sostenible se relaciona con el protagonismo que las comunidades puedan desempeñar en la definición de sus modos de vida y sus objetivos de bienestar, y, en este sentido, el papel y el marco de acción en el que se inscribirán los procesos de patrimonialización respecto a esas valoraciones. En este contexto, resulta pertinente la consideración del enfoque de capacidades inspirado en los trabajos conceptuales de Amartya Sen, de donde emerge la base filosófica de la noción de desarrollo humano.

Uno de los objetivos centrales del desarrollo humano es hacer posible que las personas se conviertan en agentes de su propia vida dentro de sus comunidades. Como afirma Sen, en las actividades del desarrollo "los individuos han de verse como seres que participan activamente -si se les da la oportunidad- en la configuración de su propio destino, no como meros receptores pasivos de los frutos de ingeniosos programas de desarrollo" (1999, p. 75). Desde esta perspectiva, el desarrollo se basa en las libertades de las personas, entendidas como oportunidades que la gente tiene para tomar decisiones y avanzar en los objetivos principales de sus vidas. Las personas deben decidir qué tipo de desarrollo desearían para sí mismas. Cuando los individuos y los grupos sociales se reconocen como agentes, pueden definir sus prioridades, así como elegir los mejores medios para alcanzarlas.

\section{DESARROLLO TERRITORIAL Y PUESTA EN VALOR DE LA DIVERSIDAD BIOCULTURAL}

Los antecedentes compartidos anteriormente respecto a la situación del ramal y su territorio dibujan un escenario en el que parece tener pertinencia el planteamiento que desarrolló hasta su cierre el programa Desarrollo Territorial con Identidad Cultural (DT-IC) de Rimisp, a saber: que en territorios que exhibían simultáneamente altos niveles de pobreza, desigualdad y discriminación, y por otro lado presentaban una dotación de patrimonio cultural y biodiversidad significativa, había una oportunidad de desarrollo territorial en el reconocimiento y puesta en valor de la identidad, la cultura y el patrimonio como vector que puede impulsar dinámicas sostenibles e inclusivas, contribuyendo a una mejor calidad de vida de poblaciones rurales latinoamericanas con menores oportunidades (Ranaboldo, 2017). Dicho de otra manera, "la diversidad $y$, en particular, las diversas manifestaciones del patrimonio cultural, son una fuente de oportunidades para procesos de desarrollo que fortalezcan las capacidades de los grupos sociales rurales y que expandan las libertades de las personas que integran las sociedades rurales de América Latina” (Berdegué, 2007, p. 3).

A partir de la experiencia del programa DT-IC y las iniciativas llevadas a cabo por los autores en ańos recientes, podemos identificar ciertas oportunidades de la puesta en valor de la diversidad biocultural en el marco de territorios con las características que ejemplifica el ramal. 
1. Cohesión social y construcción de visiones compartidas. Si entendemos el territorio como "una construcción social, es decir, un conjunto de relaciones sociales que dan origen y a la vez expresan una identidad y un sentido de propósito compartidos por múltiples agentes públicos y privados" (Schejtman y Berdegué, 2004, p. 5) podemos asumir el proceso de apropiación de los elementos que conforman el patrimonio cultural y natural de una comunidad como un factor incremental para promover la cohesión social de los actores territoriales, basado en el "interés común de proteger, valorizar y capitalizar lo que un determinado territorio tiene, sus especificidades culturales, tipicidades, naturaleza en cuanto recurso y en cuanto patrimonio ambiental, prácticas productivas y potencialidades económicas" (Albagli, 2004, en Flores, 2008, p. 39). El proceso de valorización actúa, entonces, como un procedimiento que permite transformar a los territorios locales en comunidades locales que comparten un capital social basado en el involucramiento personal, la cooperación colectiva y la autoestima individual y social, elementos con un alto potencial de innovación y de competitividad (Ledo e Iglesias, 2007).

2. Empoderamiento de actores locales. El reconocimiento y la apropiación del patrimonio biocultural puede tener una fuerte expresión psicosocial y política, que se visibiliza a través de procesos de empoderamiento de distinto nivel. Muy interesante resulta el caso de procesos de empoderamiento de mujeres indígenas rurales, las que suelen enfrentar exclusiones por la triple intersección de brechas de género, étnicas y territoriales, pero que, al mismo tiempo, son portadoras de activos culturales (saberes tradicionales, locales o comunitarios, por ejemplo, en agricultura, artesanía, gastronomía, medicina indígena o campesina, etc.) cuyo reconocimiento intra y extracomunitario ha reforzado su empoderamiento individual y grupal frente a familias, comunidades y la sociedad en general. Lo mismo puede observarse con lideresas que junto con ostentar un poder de representación política de carácter institucional, desempeñan un rol relevante en términos socioculturales. Por su parte, los procesos de empoderamiento colectivo asociados a la reivindicación de derechos culturales y territoriales tienen en el centro de sus demandas un conjunto de activos del patrimonio biocultural que son el leitmotiv de los procesos de cohesión social y otorgan un horizonte de sentido cultural y político a estos procesos y sus demandas.

3. Oportunidades económicas. De manera creciente, existe un interés y un reconocimiento relativamente extendido en estrategias de desarrollo rural asociadas a bienes y servicios con identidad territorial que ponen en valor cualidades bioculturales, estén estas basadas en una lógica de producto "ancla" o bien se inscriban en una estrategia más sistémica asociada a una canasta de productos y servicios. Estos productos y servicios ofrecen toda una gama de virtudes como aporte 
potencial a la reducción de la pobreza. En primer lugar, sus creadores son las comunidades locales, a menudo grupos pobres y marginados (mujeres, indígenas, jóvenes y afroamericanos). En segundo lugar, son productos y servicios únicos, difícilmente reproducibles, pues su calidad y valor derivan precisamente de su relación con la comunidad, a diferencia de los commodities. En tercer lugar, abren oportunidades de empleo no agrícola y, al mismo tiempo, de fortalecimiento y diversificación de habilidades, capacidades y saberes locales. En todos los casos, no se trata solo de "materias primas" sino de productos y servicios que agregan valor a los activos de una determinada población local (Flores, 2008).

Dicho en otras palabras, ya sea que el conjunto de activos del patrimonio biocultural de los territorios tenga una significación como "recurso" de actividades económicas de comunidades locales, en un lugar central o complementario de la generación de ingresos, por ejemplo a través de las actividades agropecuarias, los emprendimientos gastronómicos o la artesanía; o bien esté presente cuando las comunidades aspiran a participar del mercado turístico, diseñando ofertas basadas en su patrimonio cultural (material o inmaterial) o en el paisaje natural de sus territorios; la identidad cultural y la biodiversidad pueden constituirse en factores potenciales de estrategias de inclusión económica y productiva en territorios que cuentan con una dotación importante de estos activos.

4. Compatibilización de criterios ambientales y productivos a partir de lógicas sostenibles de acceso y uso de recursos bioculturales. Los recursos naturales y culturales propios de un territorio, y su interacción, constituyen un "activo" en la medida en que los sistemas de conocimiento de las poblaciones que los manejan, sus formas de gestión del territorio y los múltiples emprendimientos (pesca artesanal, agricultura, silvopastoreo, manejo de bosques, gastronomía, turismo, artesanías, actividades artísticas, entre otros) no sean iniciativas con un valor exclusivamente económico, sino que contribuyan al fortalecimiento de las identidades, la cohesión social local, así como las prácticas ambientalmente respetuosas.

Este enfoque, por ejemplo, resulta pertinente para las zonas de protección y conservación ambiental y sus respectivos cordones de amortiguación, territorios caracterizados muchas veces por dinámicas conflictivas y contradictorias en términos de desarrollo ambiental, social y económico, y que, a la vez, presentan una elevada concentración de biodiversidad y riqueza cultural. Muchos de estos activos constituyen la base de estrategias de vida individuales y colectivas de comunidades que habitan dichos territorios con ostensible anterioridad a la delimitación por parte de los Estados de espacios de protección de la biodiversidad. 


\section{DIÁLOGO TERRITORIAL Y NUEVOS ACUERDOS DE GOBERNANZA}

Al igual que en los conflictos socioterritoriales más recurrentes y prototípicos de nuestro continente, aunque sin grados de beligerancia significativos o preocupantes en la actualidad, y sin la connotación mediática que los caracteriza, en el caso del ramal Talca-Constitución el objeto en disputa no es el bien patrimonial ferroviario, su salvaguardia frente al estado de deterioro que presenta, las responsabilidades que le caben a la empresa en su prácticamente inexistente gestión, o al Estado en su ausente responsabilidad fiscalizadora. Como en el resto de los muchos casos en el continente, y teniendo a la vista los antecedentes de un diagnóstico que articula una mirada sistémica del espacio físico y simbólico que el tren ha construido y organizado en los últimos cien ańos, el ramal Talca-Constitución constituye un caso de conflicto socioterritorial que supone desacuerdos entre actores con intereses y prioridades diferentes sobre un determinado territorio, esto es, el objeto de disputa es el territorio, su definición, uso y significado, generalmente asociados a la estructura de propiedad, al uso y manejo de los recursos naturales, y al aprovechamiento de las oportunidades de riqueza o bienestar asociados (Fernández, 2018). Dicho de otra manera, el territorio no es solo el espacio en el que tiene lugar el conflicto, es el fundamento del mismo, la definición de un tipo de territorialidad que incluye la utilización de sus recursos y la creación o resignificación de las relaciones sociales. La definición de lo que es el territorio se convierte en el locus del conflicto (Svampa, 2008).
Muchas comunidades locales a lo largo deAmérica Latina están enfrentando este tipo de conflictos, recurriendo a diversas estrategias de defensa del territorio. En algunos casos, tal vez los menos, las estrategias de defensa y los procesos de resistencia de las comunidades derivan en la construcción de acuerdos entre los actores locales y el Estado, a partir de los cuales se gestan transformaciones institucionales y normativas que posibilitan la participación de las comunidades en la gobernanza y la gestión de ciertos espacios o recursos presentes en los territorios.

La actualización por parte de comunidades locales de estrategias no violentas de defensa del territorio, que intentan modificar las relaciones de poder en el acceso y uso de recursos naturales y culturales, presionando y negociando nuevos arreglos institucionales con los Estados nacionales, respaldados en los avances normativos internacionales como es el caso, para las comunidades indígenas, del Convenio 169 de la Organización Internacional del Trabajo (OIT) o la Declaración de los Derechos de los Pueblos Indígenas de las Naciones Unidas, ha permitido que cada vez más estos actores históricamente en situación de subalternidad puedan gestionar, en grados diversos de autonomía, bienes culturales y naturales de sus territorios. Algunas de estas iniciativas se vehiculizan a través de la puesta en valor de patrimonios culturales (tangibles e intangibles) o naturales, en esquemas de turismo comunitario; en otros casos, estas estrategias se basan en principios ambientales de protección y conservación de la biodiversidad que buscan limitar el acceso y uso indiscriminado de recursos naturales, al mismo tiempo 
que se asegura su provisión para actividades productivas, sociales y culturales, locales o ancestrales; en otros tantos se da una combinación de ambas preocupaciones y objetivos. Estos pactos y nuevos modelos de gobernanza, están lejos de constituir una solución definitiva a los conflictos territoriales de carácter ambiental e intercultural, pero representan una oportunidad significativa para poner en juego enfoques alternativos de desarrollo y de gestión del territorio, soportados en nuevos esquemas pacíficos de relación entre las comunidades locales, los Estados y otros actores extraterritoriales.

El empoderamiento y fortalecimiento de capacidades de comunidades locales a partir del reconocimiento y la apropiación de su patrimonio tanto cultural como natural permiten enriquecer los esquemas de diálogo territorial, ya sea porque elevan el grado de cohesión social fundamental para contrarrestar las diversas desigualdades (de recursos económicos, de influencia política, de información, etc.) que suelen caracterizar los procesos de diálogo con el Estado y las grandes empresas; porque colaboran en la construcción de estrategias de desarrollo alternativas para las comunidades, disputando el espacio de las "vocaciones productivas" de los territorios; o bien porque permiten orientar los procesos de inversión social tanto desde las políticas públicas como desde las empresas extractivas, por ejemplo, a través de sus procesos de responsabilidad social empresarial (RSE) o sus acciones de compensación producto de resoluciones ambientales o procesos de negociación privados.

En Chile hay algunas experiencias interesantes asociadas a nuevos esquemas insti- tucionales de gobernanza compartida entre el Estado y comunidades indígenas sobre espacios naturales protegidos. Uno de los casos más emblemáticos (El País, 2017) corresponde a los contratos de asociatividad que estableció la Corporación Nacional Forestal (Conaf), entidad pública del Estado de Chile encargada de la administración de las áreas naturales protegidas, y comunidades Licanantay de la región de Antofagasta, específicamente en la Reserva Los Flamencos (Conaf, s. f.). Las comunidades licanantay reconocen en la Reserva un territorio ancestral, en el que existen diferentes manifestaciones bioculturales, con las que la comunidad se vincula desde un punto de vista simbólico o productivo. En este contexto, el año 2002 se comienza a desarrollar un modelo de gestión asociativo, que busca responder a las demandas comunitarias por derechos de uso y participación en la administración del área protegida, a través de la gestión de proyectos turísticos y de conservación comunitarios, que han aportado a su protección y, al mismo tiempo, se han constituido en una alternativa de desarrollo local, objetivos enriquecidos por la puesta en valor del patrimonio cultural por parte de las mismas comunidades (Valenzuela, 2005)

\section{REFLEXIONES FINALES}

El caso del ramal Talca-Constitución plantea varios desafíos a propósito de los enfoques y dimensiones que hemos problematizado hasta ahora.

El primero de ellos, y del cual se derivan probablemente los otros, radica en entender, 
desde el conjunto de los actores, pero especialmente desde las comunidades y la política pública, al ramal como un territorio articulado históricamente por una vía férrea. Esta noción territorial, que concuerda con la noción de paisaje cultural para efectos del punto que queremos enfatizar, constituye la perspectiva que permite abordar, de manera consistente y coordinada, o al menos encarna dicha aspiración, los diversos procesos que comienzan a reclamar su lugar en las prioridades de los distintos actores y, particularmente, de los diversos servicios sectoriales del Estado.

Este enfoque territorial entrega, en nuestra opinión, mejores elementos de análisis y planificación a la hora de compatibilizar estrategias de patrimonialización que busquen la salvaguardia de los valores que hacen del ramal un sistema patrimonial único, que reúne expresiones tanto materiales como inmateriales, culturales y naturales -misión que inspira a los funcionarios de la institucionalidad cultural-, con las aspiraciones de mejora del ramal en tanto servicio de transporte, así como con las legítimas demandas de beneficios y oportunidades que reviertan las desigualdades socioeconómicas que afectan a las comunidades. En este sentido, el ordenamiento territorial y los procesos de zonificación de unidades dentro del paisaje constituyen herramientas de planificación territorial que pueden servir a ambos propósitos.

Un enfoque territorial, creemos, también ayudaría a visualizar estrategias más efectivas y realistas de mitigación y adaptación a las condiciones climáticas globales y su interacción con las características y manifestaciones, naturales y antrópicas, que presenta el territorio. Dicha eficacia y sus condiciones de posibilidad se relacionan directamente con la oportunidad de implicar diversos actores e intereses, locales y extraterritoriales, en el análisis de las estrategias posibles y viables. En este sentido, el enfoque territorial se aleja de nociones endogenistas que descuidan los vínculos e interacciones del territorio con otros territorios, próximos y lejanos, urbanos y rurales, con otros conocimientos, locales y expertos; $y$, por supuesto, toma en cuenta los intereses y las relaciones de poder transterritoriales que cada vez más influyen y determinan lo que se puede o no hacer en un espacio específico.

Esta complejidad que aporta la perspectiva territorial, pone de manifiesto también la necesidad de respuestas innovadoras en términos de nuevos modelos institucionales de gobernanza, flexibles y concretos, que respondan a las demandas de una ciudadanía que, por un lado, no se constituye, como en décadas pasadas, desde una homogeneidad social y cultural (de clase, de género, de etnia, etc.), y, por otro, exige formas de participación más deliberativas.

Finalmente, el desafío principal para los diversos actores involucrados es elevar al ramal Talca-Constitución a la categoría de bien común de gestión territorial -al decir de Elinor Ostrom-, cuya misión es conectar a las comunidades que une con el ejercicio de sus derechos culturales, económicos y ambientales.

\section{REFERENCIAS}

Berdegué, J. A. (2007). Presentación en C., Ranaboldo y A., Schejtman. Territorios con identidad 
cultural. Perspectivas desde América Latina y la Unión Europea. Revista Opera, (7), 1-6.

Brah, A. y Phoenix, A. (2004). Ain't I A Woman? Revisiting Intersectionality. Journal of International Women's Studies, 5(3), 75-86. Recuperado de https://vc.bridgew.edu/jiws/vol5/iss3/8

Casals, M. C. y Errázuriz, M. J. (2013). Plan Maestro Turístico. Ramal Talca-Constitución. Santiago: Ediciones Universidad Central de Chile. Recuperado de http://boletin-faup.ucentral.cl/ libro_ipt_4_de_marzo\%20.pdf

CASEN (2017). Observatorio del Ministerio de Desarrollo Social del Gobierno de Chile. Santiago: Ministerio de Desarrollo Social y de Familia.

Centro Latinoamericano para el Desarrollo Rural (RIMIsp) (2018). Relatoría Encuentro Tepoztlan (documento interno). Santiago de Chile.

Centro Latinoamericano para el Desarrollo Rural (RIMISP) (s. f.). Desarrollo territorial rural con identidad cultural. Recuperado de https://rimisp. org/proyecto/desarrollo-territorial-rural-conidentidad-cultural/

Consejo de Monumentos Nacionales de Chile (CMN) (s. f.). Santuario Inmaculada Concepción de Corinto. Santiago: CMN. Recuperado de https:// www.monumentos.gob.cl/monumentos/monumentos-historicos/santuario-inmaculadaconcepcion-corinto

Corporación Nacional Forestal (Conaf) (s. f.). Reserva Nacional Los Flamencos. Santiago: Ministerio de Agricultura, Chile en Marcha, Conaf.

El País (2017). Los moradores del desierto, abiertos a negociar. El Pais. Recuperado de https:// elpais.com/elpais/2017/08/22/planeta_futuro/1503422683_072642.html

Fernández, J. (2018). El territorio como espacio contradictorio: promesas y conflictos en torno a la actividad extractiva. Documento de Trabajo,
Rimisp-Centro Latinoamericano para el Desarrollo Rural. Santiago.

Flores, M. (2008). La identidad cultural del territorio como base de una estrategia de desarrollo sostenible. En Territorios con identidad cultural. Perspectivas desde América Latina y la Unión Europea. Revista Opera, (7)

Ledo, A. e Iglesias, M. A. (2007). La evolución del desarrollo local y la convergencia territorial. En M. Docampo García (ed.). Perspectivas teóricas en Desarrollo Local, 77-110.

Ministerio de Obras Públicas y Vivienda (2011). Estudio análisis de accesibilidad territorial - Fronteras interiores. Definición de un Plan de Accesibilidad a las zonas aisladas del territorio nacional en el periodo 2004-2010. Recuperado de http://www. koalaweb.cl/habiterra/index.php?option=com_ content\&view=article\&id=36: estudios\&catid= 28: current-users $\&$ Itemid $=44$

Morales, A. (2013). Historia del ramal Talca-Constitución: patrimonio e identidad del tren del Maule. Plan Maestro Turistico. Ramal Talca-Constitución. Santiago: Universidad Central de Chile.

Pilscoff, P. y Luebert, F. (2004). Clasificación de pisos de vegetación y análisis de representatividad ecológica de áreas propuestas para la protección en la ecorregión. Serie de Publicaciones WWF, (10). Chile: Programa Ecorregión Valdiviana.

Ranaboldo, C. (2017). Desarrollo territorial desde la identidad y el patrimonio biocultural. Rede Brasileira de Pesquisa e Gestão em Desenvolvimento Territorial-RETE.

Ranaboldo, C. y Schejtman, A. (eds.) (2009). El valor del patrimonio cultural. Territorios rurales, experiencias y proyecciones latinoamericanas. Lima: Rimisp-Centro Latinoamericano para el Desarrollo Rural, IEP. 
Schejtman, A. y Berdegué, J. A. (2004). Desarrollo Territorial Rural. Serie Debates y Temas Rurales, (1). Santiago de Chile: Rimisp-Centro Latinoamericano para el Desarrollo Rural.

Sen, A. (1999). Development as Freedom. Oxford: Oxford University Press. (traducido en castellano como Desarrollo y Libertad en 2000).

SurMaule (2015). Caracterización patrimonial del Ramal Talca-Constitución. Recuperado de http://surmaule.cl/featured_item/caracterizacion-patrimonialdel-ramal-talca-constitucion/

Svampa, M. (2008). La disputa por el territorio, movimientos de carácter socio-ambiental y discursos dominantes. Recuperado de http://maristellasvampa.net/archivos/ensayo43.pdf
Svampa, M. (2013). El Consenso de los Commodities y lenguajes de valoración en América Latina. Neoextractivismo en América Latina. Revista Nueva Sociedad, Democracia y Politica en América Latina, (244), 30-46.

Toledo, V. y Barrera-Bassols, N. (2008). La memoria biocultural: la importancia ecológica de las sabidurías tradicionales. Barcelona: Icaria Editorial.

Valenzuela, I. (2005). Modelo de gestión asociativo en la Reserva Nacional Los Flamencos. Una década de aprendizajes. Documento interno, Corporación Nacional Forestal (Conaf). Santiago: Conaf.

von Lobenstein, B. (2016). Estado y mercado en las empresas públicas. Recuperado de http://repositorio.uchile.cl/bitstream/handle/2250/139357/ Estado-y-mercado-en-las-empresas-públicas. pdf?sequence $=1$ \&isAllowed $=y$ 